$-334-$

A Comparative Study on the Power of van Lieshout and Baddeley's J-function

by

E Thönnes and $M$ van Lieshout 


\title{
A Comparative Study on the Power of Van Lieshout and Baddeley's J-function
}

\author{
Elke Thönnes \\ Department of Statistics, University of Warwick \\ Coventry CV4 7AL, UK \\ Marie-Colette van Lieshout \\ $C W I$ \\ P.O. Box 94079, 1090 GB Amsterdam, The Netherlands
}

\begin{abstract}
Summary:
Summary functions such as the empty space function $F$ and the nearest neighbour distance distribution function $G$ are often used as test statistics for point patterns. Van Lieshout and Baddeley recently proposed an alternative statistic, the $J$-function, which is defined as $J=(1-G) /(1-F)$. Theoretical advantages of the $J$-function over the $F$ - and $G$-statistics are that it measures both the type, strength and range of interaction, and that it can be evaluated explicitly for a larger class of models. In this simulation study we investigate empirically how the power of tests based on $J$ compares to that of tests based on $F$ and $G$.
\end{abstract}

1991 Mathematics Subject Classification: 60G55, 62M30, 62M11, 62G99.

Keywords and Phrases: area-interaction process, cluster process, empty space function, hard core process, J-function, Monte Carlo test, nearest neighbour distance distribution function, power.

Note: Work carried out under project PNA4.4 "Stochastic geometry".

\section{Zusammenfassung:}

Zusammenfassungsfunktionen wie die sphärische Kontaktverteilungsfunktion-Funktion $F$ und die Verteilungsfunktion des Abstandes zum nächsten Nachbarn werden häufig benutzt um Punktfelder auf komplette räumliche Zufälligkeit zu testen. Van Lieshout und Baddeley haben eine alternative Zusammmenfassungsfunktion vorgeschlagen: die $J$-funktion, die als $J=(1-G) /(1-F)$ definiert ist. Die $J$-funktion kann für mehr Modelle als die $F$ - oder die $G$-funktion analytisch berechnet werden, was ein entscheidender Vorteil ist. In dieser Simulationsstudie untersuchen wir die Stärke von Tests, die auf $J$ basieren, im Vergleich zu Tests, die auf $F$ oder $G$ basieren.

\section{INTRODUCTION}

Mapped spatial patterns arise in a variety of contexts, ranging from the positions of cell nuclei in cytology, trees in a forest, nest locations of birds or other animals to the findings of ore in material science.

The statistical analysis of such a mapped point pattern usually begins with a test for spatial randomness $[8,21]$. As a test statistic, low-dimensional summary functions such as the empty space function $F$, the nearest neighbour distance distribution function $G$ or the 
reduced second moment function $K$ are used (cf. [5, 8, 21, 22] or [24, 25]). Roughly speaking the $K$-function is proportional to the expected number of other events, that is points of the pattern, in balls of various radii around a typical event. Both $F$ and $G$ are distribution functions of distances between points: the empty space function of the distance from an arbitrary sampling point, for example the origin, to the nearest event and $G$ of the distance from an event to the nearest other event. Recently, Van Lieshout and Baddeley [13] proposed an alternative, the $J$-function, which compares inter-event distances to distances from a fixed sampling point.

In this paper we describe a simulation study which compares the power of tests based on the $J$-function with the power of tests based on $F$ and $G$. We will start by reviewing the theoretical background of $F$ and $G$ and use this to introduce the $J$-function in section 2 . Section 3 presents the models we use as alternatives to a random scattering of points and section 4 describes the simulation study. The last section discusses the results thus obtained.

\section{THEORETICAL BACKGROUND}

Throughout this paper we assume that $X$ is a stationary point process in $\mathbb{R}^{2}$. The empty space function $F$ of $X$ is then defined as

$$
F(r)=\mathbb{P}(d(0, X) \leq r) \quad r \geq 0
$$

where $d(0, X)=\min \{\|x\|: x \in X\}$, the distance from the origin to the nearest event of $X$. Similarly,

$$
G(r)=\mathbb{P}^{! 0}(d(0, X) \leq r)
$$

where $\mathbb{P}^{10}$ denotes the reduced Palm distribution of $X$. Heuristically this is the conditional distribution of the remainder $X \backslash\{0\}$ of $X$ given there is an event at the origin. For a rigorous definition see Daley and Vere-Jones [6]. Further details can also be found in [8] or [24].

In [13], the $J$-function is defined as the ratio

$$
J(r)=\frac{1-G(r)}{1-F(r)} \quad \text { for all } r \geq 0 \text { such that } F(r)<1 .
$$

It can be interpreted as the ratio of two survival functions, namely of the distance to the nearest event from (a) an event or (b) an arbitrary sampling point. Thus (2.3) compares the environment of a typical event of $X$ to the environment of an arbitrary sampling point.

If $J(r)<1$, then the survival function of (a) is smaller than that of (b) indicating clustering, whereas if $J(r)>1$, then the survival function of (b) is smaller than that of (a) indicating regularity. For a Poisson point process, $F$ and $G$ are identical and hence $J(\cdot) \equiv 1$. Thus, a single plot of the (estimated) $J$-function provides valuable information on the type and strength of interaction between events. Furtermore, it can be shown that, in contrast to $F$ and $G$, the $J$-function is constant beyond the effective range of interaction.

However, the price to pay for the lowering of dimension necessary to be able to visualise a summary statistic graphically is that neither $J$ nor $F, G$ or $K$ completely determine the distribution of $X$ and Bedford and Van den Berg give an example of a point process that is not Poisson but for which $J(\cdot) \equiv 1$. 
The $J$-function (2.3) can be expressed in terms of the conditional intensity [11] (provided it exists) which is often easier to work with than the distribution of $X$. Thus $J$ can be evaluated for a wider range of point process models than $F$ and $G$. It also behaves well under independent superposition of point patterns, a property that is very useful when studying interactions in mapped patterns consisting of different types of events.

For formal proofs and further details see [13]. Multivariate extensions of (2.3) are studied in Baddeley and Van Lieshout [14]. Plots of the $J$-function for Poisson cluster processes can be found in Saxl and Rataj [23], while Baddeley et al. consider robustness against edge effects caused by incomplete observation of $X$.

Clearly the $J$-function has many theoretical advantages over $F$ and $G$, but how does it perform empirically? In this paper we will give some answers to this question by means of simulation.

\section{The SIMUlated PROCESSES}

In order to compare the power of tests based on $J$ with tests based on $F$ and $G$ (see (2.1)(2.3)), we simulated three different types of non-Poissonian point processes: cluster processes, hard core processes and area-interaction processes. We then tested the simulated patterns against a Poisson null hypothesis and estimated the power of these tests by the proportinn of rejected patterns. In this section we will describe the simulated processes; the testing procedures used will be explained in section 4.

For all of the following processes we obtained samples on a unit square window. As all simulated processes have a finite interaction range $R$, we avoided edge effects by simulating the processes on a square window with side length $1+2 R$. Only events which are lying inside of the central unit square are used in the sample.

\subsection{Cluster process}

A cluster process $[6,17]$ is derived from a parent process by scattering a cluster of daughter points around each of the parent points. The union of all daughters then forms the cluster process. Both the random number of daughter points in each cluster as well as the locations of the daughter points relative to the parent point are independent and identically distributed. We chose a Poisson cluster process for which the parent process is a stationary Poisson process. To each parent point we assigned a number of daughter points which is a Poisson random variable of mean $\mu$. The location of the daugher points is radially normal, centred at the parent point and with standard deviation $\sigma$. The simulation of a Poisson cluster process is straightforward, for a description see [25].

\subsection{Hard core process}

In a hard core process all events are a minimum distance, the hard core distance, apart from each other. We chose a Type 2 Matérn hard core process using the algorithm described in $[15,19]$. We produced a Poisson point process and assigned an independent uniform mark to each event. If two events were closer than the hard core distance to each other we deleted the point with the smaller mark. This process leads to highly regular patterns. 


\subsection{Area-interaction process}

This process was first introduced as the penetrable spheres mixture model in [27] and then extended in [1]. It is defined by the density

$$
p(\underline{x})=\alpha \beta^{n(\underline{x})} \gamma^{-|x \oplus K|}
$$

with respect to a unit rate Poisson point process on a compact window. Here $\alpha$ is the normalizing constant and $n(\underline{x})$ is the number of events in $\underline{x}$. The parameter $\beta>0$ influences the intensity of the process. The parameter $\gamma$ does not only control the strength but also the type of interaction. For $\gamma<1$, realisations of (3.1) tend to be ordered, whereas for $\gamma>1$ clustered patterns are more likely. The strength of interaction depends on the area $|\underline{x} \oplus K|$ covered by the union of congruent compact sets $K$ centred at the events in $\underline{x}$ (using the symbol $\oplus$ for the Minkowski addition [16], i.e. $\underline{x} \oplus K=\{x+k \mid x \in \underline{x}, k \in K\}$ ). In our simulations, we chose a square for $K$.

The normalizing constant $\alpha$ in (3.1) is not computable in closed form, so we have to use a Markov Chain Monte Carlo (MCMC) method to produce samples. For an overview on MCMC methods see Gilks et al. [9]. We used the algorithm outlined in Kendall [12], coded in his $\mathrm{C}$ program Perfect. This algorithm uses the coupling from the past idea of Propp and Wilson [18] which, in contrast to conventional MCMC methods, allows for exact rather than approximate samples. For area-interaction processes with $\gamma>1$, simpler exact sampling algorithms have been developed, see Häggström et al. [10] and Thönnes [26].

\section{TESTING PROCEDURE}

\subsection{Estimation of summary statistics}

As we saw in section 2, $F$ and $G$ are defined in terms of distances between points. However, since in practice $X$ is observed within a bounded window $W$ only, inter-point distances based on $X \cap W$ for points close to the border may well differ from the 'true' distances. To deal with these edge effects, we map the point pattern onto a torus and regard the observation window as the centre of a $9 \times 9$ grid of windows with identical point patterns. The events in the other windows are taken into account when determining the nearest neighbour distances in the centre window. Note that the torus condition is only one of many possible ways to deal with edge effects [22], but in general it performs well, although it sometimes looses power for regular point patterns [20]. Surprisingly tests based on the $J$-function do not become less powerful if naive estimators which are not corrected for edge effects are used, see Baddeley et al. [2].

We estimate the summary statistics (2.1)-(2.3) as follows. For each event we determine the distance to its nearest neighbour and use the empirical cumulative distribution function of these distances as an estimator for $G$. The estimation of $F$ requires a set of sampling points, which can be a regular grid or a random set of points. We followed the recommendation in Diggle [7] and used a regular grid of sampling points with mesh size 0.1. For each of these sampling points the distance to the nearest event is determined and the empirical distribution function of these distances yields an estimator for $F$. Finally, the $J$-function is simply estimated by a ratio estimate. 


\subsection{Test statistics}

In order to define a test statistic, a measure of discrepancy between the estimated and theoretical null hypothesis values of a given summary function has to be chosen. In the simulation study below we consider a null hypothesis of complete spatial randomness (CSR), see Diggle $[7,8]$. Under this hypothesis, the point pattern is produced by a stationary Poisson process of intensity $\lambda>0$. It is easily seen that under CSR, the empty space function and the nearest neighbour distance distribution coincide and are given by

$$
F_{0}(r)=G_{0}(r)=1-\exp \left(-\lambda \pi r^{2}\right)
$$

Hence $J_{0}(r)=1$ (cf. section 2).

In the following let $H_{0}$ be the theoretical summary function under CSR and $\hat{H}_{1}$ the estimate of the appropriate summary function for the observed point pattern. If some prior knowledge about the process to be tested is available, for example its interaction radius, then it might be sufficient to use a statistic which is based on the difference between the theoretical and estimated summary function at only one specific value of $r$ :

$$
u_{1}(r)=\left|\hat{H}_{1}(r)-H_{0}(r)\right| \text {. }
$$

However, it is usually more sensible to compare values over a range of $r$. More specifically, we consider the following two test statistics.

\section{Maximum Statistic}

$$
u_{1}=\max _{0 \leq r \leq S}\left|\hat{H}_{1}(r)-H_{0}(r)\right|
$$

\section{Integral Statistic}

$$
u_{1}=\int_{0}^{S}\left(\hat{H}_{1}(r)-H_{0}(r)\right)^{2} d r
$$

Here $S$ denotes the upper limit to the range of $r$-values considered.

Hardly any distributional theory for the test statistics $u_{1}$ seems available, so we have to resort to Monte Carlo tests [3]. Thus, the value of $u_{1}$ for the data pattern is compared to values $u_{2}, \ldots, u_{m}$ obtained from $m-1$ independent simulations of the null hypothesis. The rank of $u_{1}$ then yields an exact test, since under the null hypothesis $\mathbb{P}\left(u_{1}=u_{(j)}\right)=\frac{1}{m}$, where $u_{(j)}, j \in\{1, \ldots, m\}$ denotes the $j^{\text {th }}$ order statistic. As Diggle [7] points out $m=100$ is usually sufficient since for greater $m$ the power of the test increases only marginally with $m$.

\section{RESUlts}

In the simulation study below, a Poisson null hypothesis was tested against the three alternative models discussed in section 3 . We varied the parameter settings and thus the degree of interaction for each model, tuning the overall intensity to 50 point per unit area. For each parameter setting we simulated 100 point patterns and performed the Monte Carlo tests. The number of rejected patterns yields an estimate for the power of the test.

Monte Carlo tests were performed using the Maximum and Integral Statistics (cf. section 4) on a significance level of $5 \%$. Thus 99 realisations of a Poisson point process with intensity 


\begin{tabular}{||r||r|r|r||r|r|r|}
\hline \multicolumn{1}{||c||}{} & \multicolumn{3}{c|}{ Maximum } & \multicolumn{3}{c|}{ Integral } \\
\hline S & F & G & J & F & G & J \\
\hline 0.01 & 0.05 & 0.14 & 0.13 & 0.06 & 0.13 & 0.12 \\
\hline 0.02 & 0.05 & 0.18 & 0.11 & 0.04 & 0.15 & 0.10 \\
\hline 0.03 & 0.07 & 0.20 & 0.13 & 0.07 & 0.18 & 0.14 \\
\hline 0.04 & 0.06 & 0.31 & 0.24 & 0.08 & 0.27 & 0.16 \\
\hline 0.05 & 0.11 & 0.29 & 0.22 & 0.08 & 0.30 & 0.22 \\
\hline 0.06 & 0.13 & 0.32 & 0.24 & 0.15 & 0.31 & 0.28 \\
\hline 0.07 & 0.21 & 0.27 & 0.14 & 0.17 & 0.35 & 0.31 \\
\hline 0.08 & 0.22 & 0.27 & 0.12 & 0.18 & 0.35 & 0.25 \\
\hline 0.09 & 0.25 & 0.23 & 0.03 & 0.21 & 0.30 & 0.18 \\
\hline 0.10 & 0.27 & 0.22 & 0.00 & 0.26 & 0.28 & 0.13 \\
\hline 0.11 & 0.31 & 0.22 & 0.00 & 0.28 & 0.28 & 0.05 \\
\hline 0.12 & 0.32 & 0.22 & 0.00 & 0.31 & 0.28 & 0.00 \\
\hline
\end{tabular}

Table 1: Estimated power for a cluster process with $\mu=2, \sigma=0.06$.

$\lambda=50$ were generated and the null hypothesis was rejected if the rank of $u_{1}$ was 96 or above. Regarding the range of $r$-values, recall that $J(r)(2.3)$ is only defined for $r$ such that $F(r)<1$. Simulations suggest that for our sampling scheme the probability that the estimate of $F$ becomes less than 1 is sufficiently low for values of $r$ up to 0.12 and we therefore estimated the summary functions up to range 0.12 .

\subsection{Cluster process}

First consider the Poisson cluster process described in section 3.1 with parent intensity $50 / \mu$. The degree of interaction depends on the mean number $\mu$ of daughter points and the standard deviation $\sigma$ of the normal location distribution. The more daughter points or the smaller the standard deviation $\sigma$, the more clustered the point pattern will be. This is reflected in the results summarised in tables 1-4, where the estimated power of all tests decreases with increasing standard deviation $\sigma$ or decreasing mean cluster size $\mu$.

Overall we find that for patterns with weak clustering tests based on $F$ are more powerful than tests based on $G$ and $J$, whereas for stronger clustered patterns tests based on $G$ are most powerful. We expect tests based on $G$ to be more powerful for strongly clustered patterns for the following reason. If the cluster members lie very close to each other then the distances from an event to the nearest sampling point will be approximately the same for all members of the same cluster. Thus the estimated $F$-function will be close to the $F$-function of a Poisson process with intensity 50/ $\mu$. Most nearest event-event distances on the other hand will correspond to the distance to the nearest member of the same cluster. Hence $G$ will deviate strongly from the $G$-function under CSR and we expect this deviation to be stronger than the corresponding deviation of $F$.

The $J$-function performs similar to the more powerful alternative among $F$ and $G$. For $\mu=5$ it is even the most powerful test statistic for most values of the upper bound $S$. The power of tests based on $J$ reduces considerably for large values of $S$. This is due to the 


\begin{tabular}{||r||r|r|r||r|r|r|}
\hline \multicolumn{1}{||c||}{} & \multicolumn{3}{c|}{ Maximum } & \multicolumn{3}{c|}{ Integral } \\
\hline S & F & G & J & F & G & J \\
\hline 0.01 & 0.04 & 0.26 & 0.21 & 0.07 & 0.28 & 0.21 \\
\hline 0.02 & 0.10 & 0.49 & 0.40 & 0.08 & 0.40 & 0.41 \\
\hline 0.03 & 0.07 & 0.73 & 0.64 & 0.08 & 0.65 & 0.61 \\
\hline 0.04 & 0.10 & 0.77 & 0.68 & 0.08 & 0.76 & 0.73 \\
\hline 0.05 & 0.16 & 0.75 & 0.74 & 0.11 & 0.79 & 0.80 \\
\hline 0.06 & 0.20 & 0.75 & 0.73 & 0.20 & 0.76 & 0.84 \\
\hline 0.07 & 0.31 & 0.69 & 0.62 & 0.22 & 0.78 & 0.81 \\
\hline 0.08 & 0.36 & 0.67 & 0.40 & 0.26 & 0.77 & 0.80 \\
\hline 0.09 & 0.41 & 0.64 & 0.11 & 0.36 & 0.73 & 0.72 \\
\hline 0.10 & 0.43 & 0.63 & 0.01 & 0.37 & 0.73 & 0.63 \\
\hline 0.11 & 0.44 & 0.63 & 0.00 & 0.42 & 0.73 & 0.30 \\
\hline 0.12 & 0.45 & 0.63 & 0.00 & 0.45 & 0.73 & 0.01 \\
\hline
\end{tabular}

Table 2: Estimated power for a cluster process with $\mu=2, \sigma=0.04$.

\begin{tabular}{||r||r|r|r||r|r|r|}
\hline \multicolumn{1}{||c||}{} & \multicolumn{3}{c|}{ Maximum } & \multicolumn{3}{c|}{ Integral } \\
\hline S & $\mathrm{F}$ & $\mathrm{G}$ & $\mathrm{J}$ & $\mathrm{F}$ & $\mathrm{G}$ & $\mathrm{J}$ \\
\hline 0.01 & 0.04 & 0.33 & 0.29 & 0.03 & 0.25 & 0.25 \\
\hline 0.02 & 0.05 & 0.55 & 0.47 & 0.05 & 0.47 & 0.45 \\
\hline 0.03 & 0.06 & 0.68 & 0.64 & 0.09 & 0.63 & 0.60 \\
\hline 0.04 & 0.23 & 0.77 & 0.76 & 0.13 & 0.73 & 0.76 \\
\hline 0.05 & 0.31 & 0.76 & 0.81 & 0.25 & 0.77 & 0.81 \\
\hline 0.06 & 0.45 & 0.73 & 0.81 & 0.37 & 0.80 & 0.84 \\
\hline 0.07 & 0.55 & 0.76 & 0.84 & 0.45 & 0.81 & 0.91 \\
\hline 0.08 & 0.60 & 0.76 & 0.57 & 0.55 & 0.84 & 0.93 \\
\hline 0.09 & 0.64 & 0.74 & 0.27 & 0.59 & 0.80 & 0.91 \\
\hline 0.10 & 0.71 & 0.74 & 0.01 & 0.62 & 0.79 & 0.80 \\
\hline 0.11 & 0.74 & 0.74 & 0.00 & 0.66 & 0.78 & 0.51 \\
\hline 0.12 & 0.74 & 0.74 & 0.00 & 0.71 & 0.77 & 0.08 \\
\hline
\end{tabular}

Table 3: Estimated power for a cluster process with $\mu=5, \sigma=0.06$. 


\begin{tabular}{|r||r|r|r||r|r|r|}
\hline \multicolumn{1}{||c||}{} & \multicolumn{3}{c|}{ Maximum } & \multicolumn{3}{c|}{ Integral } \\
\hline S & F & G & J & F & G & J \\
\hline 0.01 & 0.03 & 0.66 & 0.58 & 0.08 & 0.62 & 0.55 \\
\hline 0.02 & 0.01 & 0.91 & 0.87 & 0.03 & 0.82 & 0.82 \\
\hline 0.03 & 0.09 & 0.98 & 0.99 & 0.08 & 0.96 & 0.96 \\
\hline 0.04 & 0.30 & 0.99 & 0.98 & 0.18 & 0.99 & 0.99 \\
\hline 0.05 & 0.49 & 0.99 & 0.99 & 0.37 & 0.99 & 1.00 \\
\hline 0.06 & 0.61 & 0.99 & 1.00 & 0.50 & 0.99 & 1.00 \\
\hline 0.07 & 0.73 & 0.98 & 1.00 & 0.67 & 1.00 & 1.00 \\
\hline 0.08 & 0.75 & 0.98 & 0.90 & 0.71 & 1.00 & 1.00 \\
\hline 0.09 & 0.80 & 0.98 & 0.48 & 0.73 & 0.99 & 1.00 \\
\hline 0.10 & 0.83 & 0.98 & 0.02 & 0.77 & 0.99 & 1.00 \\
\hline 0.11 & 0.84 & 0.98 & 0.00 & 0.80 & 0.99 & 0.90 \\
\hline 0.12 & 0.85 & 0.98 & 0.00 & 0.83 & 0.97 & 0.33 \\
\hline
\end{tabular}

Table 4: Estimated power for a cluster process with $\mu=5, \sigma=0.04$.

fact that the variance of the estimator for $J(r)$ increases drastically for large values of $r$ because the estimated $F(r)$ approaches 1. This is more noticable for the Maximum Statistic than for the Integral Statistic as the latter is more robust and hence less affected by random fluctuations.

\subsection{Hard core process}

Our second results concern the Type 2 Matérn hard core process introduced in section 3.2. For this model, the greater the hard core distance $h$, the more regular are the patterns produced by the process.

The results are summarised in tables 5-6. It can be seen that when $h$ increases, the power of all tests considered increases as well. For the hard core process, generally tests based on the $F$-function are least powerful. Tests based on $G$ and $J$ are similar in power; the $J$-function is slighly more powerful, in particular if the Integral statistic is used.

As in the case of cluster process alternatives, some decrease in power is noticable for $J$ based tests when $S$ gets large, but the effect is much less pronounced. As mentioned before the loss of power is due to the increasing variance of the estimate of $J$ both for the tested pattern as for the Poisson point patterns. The variance for the estimate of $J$ is bounded for clustered processes as the estimate will take values between 0 and 1 . In contrast there is no such bound for Poisson point patterns or hard core patterns. Thus due to averaging effect the decrease in power when testing hard core patterns is smaller than when testing cluster processes. 


\begin{tabular}{||r||r|r|r||r|r|r|}
\hline \multicolumn{1}{||c||}{} & \multicolumn{3}{c|}{ Maximum } & \multicolumn{3}{|c|}{ Integral } \\
\hline S & $\mathrm{F}$ & $\mathrm{G}$ & $\mathrm{J}$ & $\mathrm{F}$ & $\mathrm{G}$ & $\mathrm{J}$ \\
\hline 0.01 & 0.05 & 0.00 & 0.01 & 0.04 & 0.00 & 0.00 \\
\hline 0.02 & 0.04 & 0.00 & 0.04 & 0.02 & 0.00 & 0.04 \\
\hline 0.03 & 0.03 & 0.06 & 0.30 & 0.04 & 0.00 & 0.17 \\
\hline 0.04 & 0.05 & 0.83 & 0.80 & 0.03 & 0.64 & 0.57 \\
\hline 0.05 & 0.04 & 0.81 & 0.69 & 0.05 & 0.81 & 0.72 \\
\hline 0.06 & 0.05 & 0.60 & 0.50 & 0.05 & 0.65 & 0.68 \\
\hline 0.07 & 0.02 & 0.40 & 0.36 & 0.03 & 0.49 & 0.54 \\
\hline 0.08 & 0.02 & 0.34 & 0.30 & 0.03 & 0.42 & 0.49 \\
\hline 0.09 & 0.01 & 0.31 & 0.24 & 0.02 & 0.33 & 0.35 \\
\hline 0.10 & 0.01 & 0.30 & 0.17 & 0.03 & 0.29 & 0.28 \\
\hline 0.11 & 0.01 & 0.29 & 0.16 & 0.03 & 0.27 & 0.25 \\
\hline 0.12 & 0.01 & 0.29 & 0.10 & 0.02 & 0.24 & 0.19 \\
\hline
\end{tabular}

Table 5: Estimated power for a hard core process with $h=0.04$.

\begin{tabular}{||r||r|r|r||r|r|r|}
\hline \multicolumn{1}{||c||}{} & \multicolumn{3}{c|}{ Maximum } & \multicolumn{3}{c|}{ Integral } \\
\hline S & F & G & J & F & G & J \\
\hline 0.01 & 0.08 & 0.00 & 0.03 & 0.09 & 0.00 & 0.00 \\
\hline 0.02 & 0.04 & 0.00 & 0.06 & 0.05 & 0.00 & 0.00 \\
\hline 0.03 & 0.03 & 0.06 & 0.27 & 0.05 & 0.00 & 0.20 \\
\hline 0.04 & 0.07 & 0.80 & 0.79 & 0.05 & 0.61 & 0.59 \\
\hline 0.05 & 0.14 & 0.89 & 0.99 & 0.08 & 0.88 & 0.96 \\
\hline 0.06 & 0.13 & 0.90 & 1.00 & 0.11 & 0.90 & 1.00 \\
\hline 0.07 & 0.19 & 0.92 & 1.00 & 0.14 & 0.90 & 1.00 \\
\hline 0.08 & 0.21 & 0.91 & 0.96 & 0.15 & 0.90 & 0.99 \\
\hline 0.09 & 0.19 & 0.91 & 0.89 & 0.19 & 0.90 & 0.97 \\
\hline 0.10 & 0.19 & 0.91 & 0.74 & 0.17 & 0.91 & 0.92 \\
\hline 0.11 & 0.19 & 0.91 & 0.67 & 0.16 & 0.91 & 0.87 \\
\hline 0.12 & 0.19 & 0.91 & 0.42 & 0.13 & 0.91 & 0.72 \\
\hline
\end{tabular}

Table 6: Estimated power for a hard core process with $h=0.06$. 


\begin{tabular}{||r||r|r|r||r|r|r|}
\hline \multicolumn{1}{||c||}{} & \multicolumn{3}{c|}{ Maximum } & \multicolumn{3}{|c|}{ Integral } \\
\hline S & F & G & J & F & G & J \\
\hline 0.01 & 0.03 & 0.00 & 0.00 & 0.04 & 0.01 & 0.00 \\
\hline 0.02 & 0.04 & 0.00 & 0.01 & 0.03 & 0.00 & 0.00 \\
\hline 0.03 & 0.06 & 0.03 & 0.07 & 0.06 & 0.00 & 0.03 \\
\hline 0.04 & 0.07 & 0.24 & 0.24 & 0.06 & 0.20 & 0.12 \\
\hline 0.05 & 0.06 & 0.38 & 0.38 & 0.06 & 0.31 & 0.29 \\
\hline 0.06 & 0.04 & 0.40 & 0.53 & 0.07 & 0.43 & 0.50 \\
\hline 0.07 & 0.09 & 0.50 & 0.59 & 0.03 & 0.51 & 0.66 \\
\hline 0.08 & 0.08 & 0.52 & 0.61 & 0.05 & 0.57 & 0.65 \\
\hline 0.09 & 0.06 & 0.51 & 0.59 & 0.06 & 0.55 & 0.69 \\
\hline 0.10 & 0.06 & 0.50 & 0.56 & 0.07 & 0.55 & 0.66 \\
\hline 0.11 & 0.06 & 0.50 & 0.47 & 0.07 & 0.53 & 0.61 \\
\hline 0.12 & 0.06 & 0.50 & 0.32 & 0.06 & 0.50 & 0.49 \\
\hline
\end{tabular}

Table 7: Estimated power for an area-interaction process with $g=-200, k=0.1$.

\subsection{Area-interaction process}

Two parameters influence the strength of the interaction in an area-interaction process, the parameter $\gamma$ and $k$, the sidelength of the square $K$. If $g$ denotes the logarithm of $\gamma$, then for $g<0$ the model exhibits repulsion, whereas $g>0$ will lead to aggregation.

The density of the area-interaction process weighs a Poisson process according to an exponential of the area of $\underline{x} \oplus K$, which is a functional of the empty space. Thus it seems surprising that tests based on the empty space function $F$ are the least powerful, both in the repulsive as in the attractive case. However if we increase (respectively decrease) the area of $\underline{x} \oplus K$, the distance between the points of $\underline{x}$ will increase (decrease) overproportionally, which explains why tests based on $G$ are more powerful for area-interaction processes. The power of tests based on $J$ is similar to that of tests based on $G$, see tables 7-10.

Repulsive area-interaction From tables 7 and 8 , in the repulsive case the power of tests based on $J$ is slightly better than that of tests based on $G$.

Attractive Area-Interaction In the attractive case the power of tests based on $J$ is similar to the power of tests based on $G$, see tables 9 and 10 . However, as for cluster processes, the power of $J$-based tests reduces considerably if the upper limit $S$ of the Integral and Maximum Statistics is increased.

\section{SUMMARY}

Overall we found that using the $J$-function to test for CSR is a competitive alternative to $G$ and $F$. The $J$-function produces tests which are of similar power as the more powerful of the alternatives $F$ and $G$. For repulsive processes tests based on $J$ are often slightly more powerful than tests based on the alternative summary functions. However, the performance of $J$-based tests considerably worsens as the range of values taken into account grows, especially 


\begin{tabular}{||r||r|r|r||r|r|r|}
\hline \multicolumn{1}{||c||}{} & \multicolumn{3}{c|}{ Maximum } & \multicolumn{3}{|c|}{ Integral } \\
\hline S & F & G & J & F & G & J \\
\hline 0.01 & 0.02 & 0.00 & 0.01 & 0.03 & 0.00 & 0.01 \\
\hline 0.02 & 0.05 & 0.00 & 0.03 & 0.05 & 0.00 & 0.02 \\
\hline 0.03 & 0.04 & 0.03 & 0.12 & 0.04 & 0.00 & 0.05 \\
\hline 0.04 & 0.06 & 0.47 & 0.25 & 0.05 & 0.37 & 0.21 \\
\hline 0.05 & 0.06 & 0.59 & 0.59 & 0.04 & 0.57 & 0.41 \\
\hline 0.06 & 0.06 & 0.74 & 0.68 & 0.03 & 0.72 & 0.66 \\
\hline 0.07 & 0.10 & 0.78 & 0.75 & 0.04 & 0.80 & 0.78 \\
\hline 0.08 & 0.11 & 0.79 & 0.77 & 0.04 & 0.81 & 0.82 \\
\hline 0.09 & 0.07 & 0.78 & 0.76 & 0.06 & 0.81 & 0.82 \\
\hline 0.10 & 0.05 & 0.77 & 0.71 & 0.06 & 0.82 & 0.80 \\
\hline 0.11 & 0.05 & 0.77 & 0.59 & 0.06 & 0.82 & 0.77 \\
\hline 0.12 & 0.05 & 0.76 & 0.45 & 0.04 & 0.81 & 0.67 \\
\hline
\end{tabular}

Table 8: Estimated power for an area-interaction process with $g=-300, k=0.1$.

\begin{tabular}{||r||r|r|r||r|r|r|}
\hline \multicolumn{1}{|c||}{} & \multicolumn{3}{c|}{ Maximum } & \multicolumn{3}{c|}{ Integral } \\
\hline S & F & G & J & F & G & J \\
\hline 0.01 & 0.04 & 0.28 & 0.27 & 0.04 & 0.28 & 0.24 \\
\hline 0.02 & 0.04 & 0.46 & 0.38 & 0.05 & 0.42 & 0.33 \\
\hline 0.03 & 0.05 & 0.65 & 0.53 & 0.06 & 0.60 & 0.52 \\
\hline 0.04 & 0.04 & 0.66 & 0.56 & 0.05 & 0.66 & 0.61 \\
\hline 0.05 & 0.04 & 0.61 & 0.53 & 0.06 & 0.69 & 0.64 \\
\hline 0.06 & 0.13 & 0.58 & 0.53 & 0.06 & 0.66 & 0.68 \\
\hline 0.07 & 0.20 & 0.56 & 0.34 & 0.12 & 0.65 & 0.67 \\
\hline 0.08 & 0.26 & 0.54 & 0.15 & 0.18 & 0.60 & 0.56 \\
\hline 0.09 & 0.24 & 0.46 & 0.03 & 0.20 & 0.57 & 0.40 \\
\hline 0.10 & 0.26 & 0.46 & 0.00 & 0.26 & 0.53 & 0.28 \\
\hline 0.11 & 0.29 & 0.46 & 0.00 & 0.28 & 0.51 & 0.07 \\
\hline 0.12 & 0.30 & 0.46 & 0.01 & 0.30 & 0.50 & 0.02 \\
\hline
\end{tabular}

Table 9: Estimated power for an area-interaction process with $g=180, k=0.1$. 


\begin{tabular}{||r||r|r|r||r|r|r|}
\hline \multicolumn{1}{||c||}{} & \multicolumn{3}{c|}{ Maximum } & \multicolumn{3}{|c|}{ Integral } \\
\hline S & F & G & J & F & G & J \\
\hline 0.01 & 0.05 & 0.38 & 0.37 & 0.08 & 0.39 & 0.32 \\
\hline 0.02 & 0.07 & 0.70 & 0.66 & 0.07 & 0.67 & 0.60 \\
\hline 0.03 & 0.08 & 0.82 & 0.78 & 0.09 & 0.78 & 0.72 \\
\hline 0.04 & 0.15 & 0.87 & 0.91 & 0.10 & 0.86 & 0.84 \\
\hline 0.05 & 0.19 & 0.90 & 0.90 & 0.15 & 0.90 & 0.90 \\
\hline 0.06 & 0.25 & 0.92 & 0.91 & 0.16 & 0.93 & 0.95 \\
\hline 0.07 & 0.36 & 0.88 & 0.82 & 0.28 & 0.91 & 0.94 \\
\hline 0.08 & 0.44 & 0.87 & 0.58 & 0.34 & 0.92 & 0.94 \\
\hline 0.09 & 0.47 & 0.85 & 0.17 & 0.41 & 0.87 & 0.89 \\
\hline 0.10 & 0.51 & 0.85 & 0.00 & 0.44 & 0.85 & 0.74 \\
\hline 0.11 & 0.54 & 0.85 & 0.00 & 0.49 & 0.84 & 0.43 \\
\hline 0.12 & 0.56 & 0.84 & 0.00 & 0.55 & 0.83 & 0.09 \\
\hline
\end{tabular}

Table 10: Estimated power for an area-interaction process with $g=250, k=0.1$.

when using the Maximum Statistic. This is due to the fact that the $J$-function is a ratio and the variance of its estimator increases as the denominator approaches zero. The loss of power is more pronounced for processes with attractive interaction. Because of these observations, we would recommend to consider only those ranges for which the empty spaice function is sufficiently below 1 and to use the Integral Statistic, which is less affected by sampling fluctuations.

\section{ACKNOWLEDGEMENT}

We are grateful to Wilfrid Kendall for valuable suggestions and access to his code for simulating the area-interaction process. This research was partially performed when Marie-Colette van Lieshout was at the University of Warwick and supported by the Nuffield Foundation grant SCI / 180/94/103. Elke Thönnes was supported by an EPSRC earmarked studentship and a University of Warwick graduate award. Some of this research was performed during a visit of the first author at CWI; its hospitality and financial support are gratefully acknowledged. 


\section{References}

1. BAdDeley, A.J. ANd Lieshout, M.N.M. VAN (1995) Area-interaction point processes. Annals of the Institute of Statistical Mathematics 47, 601-619.

2. Baddeley, A.J., Kerscher, M., Schladitz, K. ANd Scott, B.T.(1997) Estimating the J function without egde correction. Research Report of the Department of Mathematics, University of Western Australia, 1997/25.

3. Besag, J. AND Diggle, P.J. (1977) Simple Monte Carlo tests for spatial pattern. Applied Statistics 26, 327-333.

4. BEDFORD,T. AND BERG, J. VAN DEN (1997) A remark on Van Lieshout and Baddeley's J-function for point processes. Advances in Applied Probability (SGSA) 29, 19-25.

5. Cressie, N.A.C. (1993) Statistics for spatial data. John Wiley \& Sons, New York, revised edition.

6. DALEY, D.J. AND VerE-JONES, D. (1988) An introduction to the theory of point processes. Springer-Verlag, New York.

7. DigGLE, P.J. (1979) On parameter estimation and goodness-of-fit testing for spatial point patterns. Biometrics 35, 87-101.

8. Diggle, P.J. (1983) Statistical analysis of spatial point patterns. Academic Press, London.

9. Gilks, W.R., Richardson, S. AND SPIEgelhalteR, D.J. (1996) Markov Chain Monte Carlo in practice. Chapman \& Hall, London.

10. HägGströM, O., LIEshout, M.N.M VAN AND MøLleR, J. (1998) Characterisation results and Markov Chain Monte Carlo algorithms including exact simulation for some spatial point processes. Bernoulli, to appear.

11. Kallenberg, O. (1984) An informal guide to the theory of conditioning in point processes. International Statistical Review 52, 151-164. 
12. Kendall, W.S. (1998) Perfect simulation for the area-interaction point process. Proceedings of the Symposium on Probability towards the Year 2000. Edited by L. Arccardi and C. Heyde. Springer-Verlag, Berlin, to appear.

13. LIESHOUT, M.N.M VAN AND BADDELEY, A.J. (1996) A nonparametric measure of spatial interaction in point patterns. Statistica Neerlandica 3, 344-361.

14. LIEShOUT, M.N.M. VAN AND BADDELEY, A.J. (1998) Indices of dependence between types in multivariate point patterns. Scandinavian Journal of Statistics, to appear.

15. MATÉRN, B. (1960) Spatial variation. Meddelanden fran statens Skogsforskningsinstitut 49(5), 1-141.

16. Matheron, G (1975) Random sets and integral geometry. John Wiley \& Sons, New York.

17. NeYMAN, J. AND SCOTT, E.L. (1958) Statistical approach to problems of cosmology. Journal of the Royal Statistical Society B 20, 1-43.

18. Propp, J.G. AND WILsON, D.B. (1996) Exact sampling with coupled Markov chains and applications to statistical mechanics. Random Structures and Algorithms 9, 223252.

19. RIPLEY, B.D. (1977) Modelling spatial patterns. Journal of the Royal Statistical Society B 39, 172-212.

20. RIPLEY, B.D. (1979) Tests of randomness for spatial point pattern. Journal of the Royal Statistical Society B 41, 368-374.

21. RIPLEY, B.D. (1981) Spatial statistics. John Wiley \& Sons, New York.

22. RIPLEY, B.D. (1988) Statistical inference for spatial processes. Cambridge University Press, Cambridge.

23. SAXL, I. AND RATAJ, J. (1996) Spherical contact and nearest-neighbour distances in Boolean cluster fields. Acta Stereologica 15(1), 91-96.

24. Stoyan, D., Kendall, W.S. AND Mecke, J. (1995) Stochastic geometry and its applications. John Wiley \& Sons, Chichester, Second Edition.

25. Stoyan, D. AND Stoyan, H. (1994) Fractals, random shapes and point fields. John Wiley \& Sons, Chichester.

26. THÖNNES, E. (1998) Perfect Simulation of some point processes for the impatient user. Advances in Applied Probability (SGSA), to appear.

27. WIDOM, J.S. AND ROWLINSON, B. (1970) New model for the study of liquid-vapour phase transitions. Journal of Chemical Physics 52, 1670-1684. 